\title{
Generation of scalp discharges in temporal lobe epilepsy as suggested by intraoperative electrocorticographic recordings
}

\author{
J L Fernández Torre, G Alarcón, C D Binnie, J J García Seoane, J Juler, C N Guy, \\ C E Polkey
}

Institute of Epileptology, King's College Hospital, Denmark Hill, London, UK J L Fernández Torre G Alarcón

C D Binnie

J Juler

C E Polkey

Departamento de Fisiología, Facultad de Medicina, Universidad Complutense

Madrid 28040, Spain

G Alarcón

J J García Seoane

Imperial College of Science, Technology and Medicine, Physics Department

(Biophysics Group), The Blackett Laboratory, Prince Consort Road, London, UK C N Guy

Correspondence to: Dr Gonzalo Alarcón Department of Clinical Neurophysiology, King's College Hospital, Denmark Hill, London SE5 9RS, UK. Telephone 0044171346 5309; fax 0044171346 5308

Received 14 September 1998 and in revised form 28 January 1999

Accepted 4 February 1999
Abstract

Objectives-To study the variability, topography, polarity, duration, and incidence of interictal epileptiform discharges (EDs) in the scalp EEG and electrocorticogram (ECoG) from 16 patients with temporal lobe epilepsy who underwent surgical treatment.

Methods-Preoperative scalp EEGs during quinalbarbitone induced sleep were compared with preresection ECoGs obtained under general anaesthesia. The analysis was based on the initial ECoG record obtained before activation by intravenous thiopentone, and the EEG during stages I and II of sleep.

Results-On the scalp, 15 patients had a single discharge pattern, spikes were predominantly negative, EDs were of largest amplitude at the anterior temporal electrode in 13 patients and mean discharge incidence was 4.0 (SD 4.2) discharges/ min. In ECoG recordings, nine patients had two independent ECoG patterns, the polarity of spikes was negative, positivenegative, or positive, the site of maximal amplitude varied greatly between subjects, discharge incidence was 7.3 (SD 3.9) discharges $/ \mathrm{min}$. There was no relation between the topography of the largest spikes on the scalp and in the ECoG. In 14 patients, scalp spikes showed statistically significant longer duration on the scalp than in the ECoG. In seven patients who had frequent widespread ECoG discharges, averaging spikes across ECoG channels generated spiky patterns of duration similar to that of scalp spikes.

Conclusion-It seems that, in temporal lobe epilepsy, scalp discharges originate from widespread ECoG discharges and tend to produce a stereotyped pattern on the scalp with largest amplitudes at the anterior temporal electrodes. This is probably due to local anatomical peculiarities in the brain coverings, such as skull discontinuities, rather than to the location of neuronal generators within the temporal lobe. Due to spatiotemporal averaging, widespread cortical discharges which become asynchronous during propagation appear with increased duration and blunted waveform in the EEG, whereas sharply localised phenomena such as positive focal spikes are not recorded from the scalp.

(F Neurol Neurosurg Psychiatry 1999;67:51-58)
Keywords: scalp electroencephalography; electrocorticography; temporal lobe epilepsy; epileptiform discharge

The topography of interictal epileptiform discharges recorded on the scalp is often evaluated for the identification and classification of epileptic syndromes. In partial epilepsies, the spatial distribution of discharges is considered an important criterion to identify the lobe from where seizures arise, both to establish a syndromic diagnosis and for presurgical assessment. Several methods have been developed to estimate the origin of epileptiform discharges from scalp recordings but the principles involved in the transmission of electric fields through the brain and its coverings are largely unknown. For instance, epileptiform discharges in temporal lobe epilepsy often show the largest amplitude at the inferior frontal electrode, which is located closer to frontal than to temporal structures. ${ }^{1}$ Intracranial EEG recordings are often required in the evaluation of patients for epilepsy surgery to overcome the ambiguities of scalp recordings. It is, however, surprising how different intracranial epileptiform discharges can be from discharges recorded on the scalp. Although both are generated by the same pathophysiological phenomena, the electrode locations, the distance of electrodes from biological generators and, particularly, the intervening tissues are markedly different. It seems that intracranial discharges are more frequent, more variable, shorter in duration, and sharper than discharges recorded on the scalp. Intracranial discharges often show more complex topographic patterns than scalp discharges. ${ }^{2-4}$ In addition, whereas scalp discharges are predominantly negative, positive as well as negative polarities and dipolar configurations can be seen on intracranial records. Such discrepancies between scalp and intracranial recordings can only partially be explained by the fact that not all intracranial discharges are seen on the scalp ${ }^{5}$ or on the basis of differences in recording distance and electrode type or location. These discrepancies have been anecdotally reported by several authors, ${ }^{2-5}$ but a rigorous comparison between intracranial and scalp recordings has been hindered by the fact that intracranial recordings have not always included a sufficient coverage of a cortical region to be compared with the overlying scalp. When they do, the craniotomy wound or burr holes, damage to brain coverings, and the intracranial electrodes 
themselves preclude any simultaneous scalp recording typical of the patient's habitual EEG.

Interictal scalp EEGs are nearly always obtained before surgery, to estimate the side and lobe from where seizures are likely to arise. Intraoperative electrocorticography $(\mathrm{ECoG})$ is commonly performed to evaluate the extent of interictal epileptiform discharges as a guide to the surgical procedure. Both methods provide detailed topographical and temporal characterisation of electric fields, allowing the study of the relation between scalp and intracranial recordings. Surprisingly, the comparison between scalp and ECoG recordings has received little attention in the past, although it can answer some fundamental questions about how electric fields are transmitted through the brain coverings. As corticographic records are more precise estimators of the topography of neuronal generators, establishment of corticographic correlates of scalp phenomena could be used to explain the generation of discharges on the scalp and should aid the interpretation of scalp recordings. We have studied the variability, topography, polarity, duration, and frequency of interictal epileptiform discharges in scalp and ECoG records from 16 patients with refractory temporal lobe epilepsy who underwent surgical treatment. Corticographic findings with regard to these variables have been correlated with scalp recordings.

\section{Material and methods}

PATIENTS

Sixteen patients were studied, six female and 10 male, aged between 7 and 52 years (mean 23.3 (SD 11.49). They were considered for resective temporal lobe surgery because of medically intractable complex partial or secondarily generalised seizures. They were investigated according to the local protocol. ${ }^{6}$

RECORDING OF SCALP EEG AND ECoG

Scalp EEGs were recorded in all patients, including a waking record with hyperventilation and photic stimulation and a recording during sleep induced by oral quinalbarbitone (100-200 mg). The Maudsley electrode placement system ${ }^{7}$ was used. This system includes anterior temporal placements, which are located about $15 \mathrm{~mm}$ anterior to the $\mathrm{T} 1$ and $\mathrm{T} 2$ locations of the extended $10 / 20$ system $^{8}$ and will be designated $\mathrm{T} 1$ and $\mathrm{T} 2$ here. ECoGs were performed under general anaesthesia with nitrous oxide and isoflurane using an array of up to 21 saline wick electrodes independently placed over the superior, middle, and inferior temporal gyrus, and suprasylvian cortex. One monopolar flexible electrode was inserted under the orbitofrontal region and four similar electrodes were inserted along the subtemporal cortex. For the analysis, subtemporal electrodes were grouped into anterior, middle (two electrodes), and posterior. Common average reference recording was used in all ECoG records. The EEG records were obtained in common average and in bipolar montages. Both EEG and ECoG were recorded using an analog 21 channel Nihon Kohden electroencephalograph with a high frequency filter at 70
$\mathrm{Hz}$ and time constant at 0.3 seconds. In addition, ECoG records from 15 patients were digitised at $166 \mathrm{~Hz}$. The analysis was based on the initial ECoG record obtained under general anaesthesia before activation by intravenous thiopentone, and the EEG during stages I and II of sleep.

\section{TERMINOLOGY}

Throughout the paper, the term spike will be used to describe a sharp transient waveform seen in any recording channel (including sharp waves according to the terminology of the International Federation of Societies for Electroencephalography and Clinical Neurophysiology). ${ }^{9}$ The term discharge will be used to describe a set of spikes recorded at different sites within a limited time span. ${ }^{10}$ In the present study, spikes were considered to belong to the same discharge if they occurred within $500 \mathrm{~ms}$. For each record, discharges were classified into patterns according to the topography of the set of spikes associated with the discharge. A pattern was defined by the topography of the most widespread discharge involving a particular set of electrodes. Any discharge more restricted was considered to belong to the same pattern if the electrodes were a subset of those involved in the more widespread discharge. This concept of pattern was applied to scalp and to ECoG recordings. For each discharge pattern, and for individual discharges, we defined a dominant electrode as the electrode that recorded largest voltage changes, identified by spikes of maximal amplitude in a referential montage or phase reversal on a bipolar montage. More than one dominant electrode could be defined for a given pattern if not all individual discharges belonging to it showed maximal amplitude at the same electrode. Thus each individual discharge will show only one dominant electrode, whereas patterns may have more than one dominant electrode.

\section{METHODS OF ANALYSIS}

Scalp recordings

Epileptiform discharges for each patient were identified in the preoperative scalp EEG by spikes recorded on the operated side. In each record the incidence and topography of discharges were determined during two periods of time equal to the preactivation period in the ECoG of the same patient. To maintain conditions as far as possible equivalent to ECoG obtained during light general anaesthesia, the assessment was performed during stages I and II of sleep. In addition, the duration of scalp discharges was defined by the duration of the spike seen at the dominant electrode with a common average reference recording. The duration of all discharges, seen in the periods of the paper trace recorded in common reference derivation, was measured.

\section{ECo $G$ recordings}

The different discharge patterns present in ECoG recordings were identified for each patient. The number of discharges recorded for each pattern during the preactivation time was counted and the incidence of each pattern was 

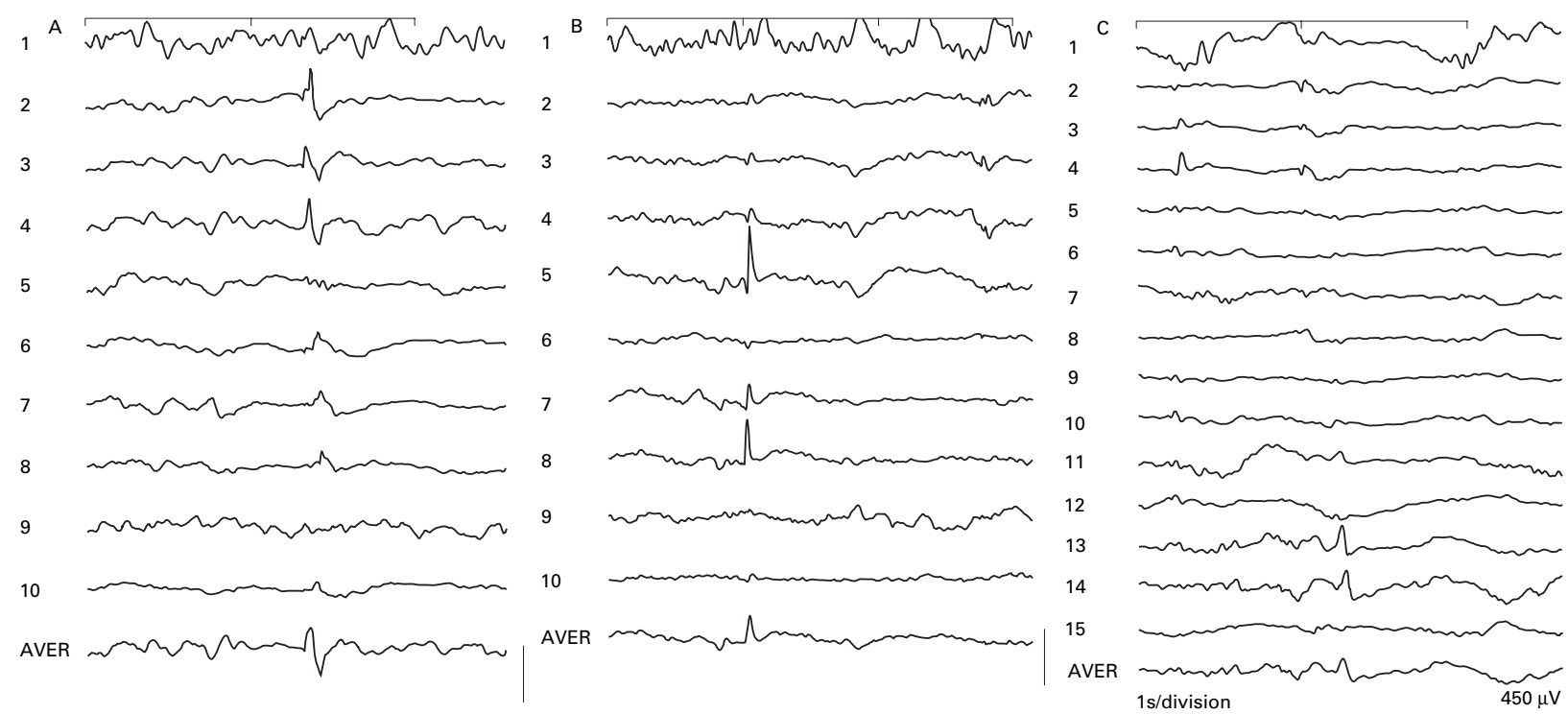

Average of intracranial channels showing sharp spikes with small latency differences generates a waveform which is blunter and has longer duration than individual intracranial spikes. (A) An epileptiform discharge involving the subtemporal cortex. $1=$ orbital; 2, 3, 4, 5=subtemporal (from anterior to posterior); $6,7,8,9=$ inferior temporal gyrus (from anterior to posterior); $10=$ midtemporal gyrus; $A V E R=$ average of channels 2,3 , and 4 . (B) $A$ lateral temporal discharge from the same patient. Electrode positions as in A. AVER=average of channels 5, 7, and 8. (C) A lateral temporal discharge from another patient. $1=$ orbital; 2, 3, 4, 5, 6, 7=subtemporal (from anterior to posterior); 8, 9, 10, 11=inferior temporal gyrus (from anterior to posterior); 12 , 13, 14=midtemporal gyrus (from anterior to posterior); 15=superior temporal gyrus; AVER=average of channels 13 and 14 .

expressed in discharges/min. For purposes of analysis, electrodes were grouped into subtemporal, lateral temporal, suprasylvian, and orbital. Subtemporal electrodes were further classified into anterior, mid, and posterior. Lateral temporal electrodes were divided into superior, mid, and inferior temporal gyrus. Each gyrus was further divided into anterior and posterior. Spikes were classified according to their polarity into: negative spikes, positive spikes and positive-negative spikes (usually, but not always, showing an initial low amplitude positive deflection followed by a larger amplitude negative spike). The duration was defined for each discharge as the duration of the broadest (longest lasting) spike detected in any channel. In negative (or positive) spikes, spike duration was measured between the beginning of the ascending (or descending, if spikes were positive) flank and the point at the same voltage on the following flank. In positive-negative spikes, spike duration was measured between the ascending flank at baseline voltage and the point at the same voltage on the following flank.

\section{Comparison of the duration of scalp and ECoG} discharges

For each patient, means of durations of scalp and $\mathrm{ECoG}$ discharges were compared using an unpaired two tailed Student's $t$ test. Differences were assumed to be significant if $\mathrm{p}<0.05$. Before applying the Student's $t$ test, the presence of equal or unequal variances was examined by Levene's test.

\section{Spatial averaging}

Digitised ECoG discharges involving two or more channels were averaged off line in an attempt to simulate the effects of spatiotemporal blurring on the duration and morphology of scalp discharges for the same patient. For each discharge, channels showing spikes were aver- aged sample by sample (figure). This average was regarded as a model of the scalp record. The duration of modelled spikes which showed polarity similar to scalp spikes was measured as described above. The means of the durations measured in spikes recorded on the scalp, and in spikes modelled from ECoG discharges, were compared using a Student's $t$ test for unpaired samples. Again differences were assumed to be significant if $\mathrm{p}<0.05$ and before comparing means by Student's $t$ test, the presence of equal or unequal variances was examined by Levene's test. Modelling and statistical analysis were performed in all patients who met the above criteria (discharges involving more than one electrode with polarity similar to scalp discharges in the same patient) in at least five ECoG spikes.

\section{Results}

ANALYSIS OF SCALP DISCHARGES

Only discharges ipsilateral to the side of surgery were analysed. These were present in all patients. Contralateral discharges were present in six patients $(37.5 \%)$ but were not considered, as there were no ECoG recordings on the contralateral side. Fifteen patients had a single discharge pattern on the scalp and one patient had two independent scalp discharge patterns. The patient who had two discharge patterns showed some discharges with spikes restricted to the superior frontal electrode (F3) and independent discharges involving $\mathrm{T} 1, \mathrm{~A} 1$, T3, and T5 with largest amplitude at A1. Among the 15 patients showing a single discharge pattern on the scalp, the dominant electrodes were anterior temporal (T1/T2) in 13 patients, midtemporal (A1/A2) in four, posterior temporal (T5/T6) in two patients, and sylvian electrode (T3/T4) in one patient. Five of these 15 patients showed two dominant electrodes: in three patients the anterior 
temporal (T1/T2) and the midtemporal (A1/ A2) were the dominant electrodes, in one patient the midtemporal (A1/A2) and the posterior temporal electrodes (T5/T6) were dominant, and in one patient the midtemporal (A1/ A2) and sylvian electrodes (T3/T4) were dominant. When considering all 16 patients, the prefrontal electrodes (Fp1/Fp2) were involved in the discharges of 11 patients, either consistently in all discharges (seven patients) or occasionally (four patients). In only one patient the anterior temporal electrode was not involved in the discharges and in two further patients the anterior temporal electrodes were involved in the discharges but were nondominant. Among all 16 patients, the midtemporal electrodes were involved in the discharges but were non-dominant in 10 patients, the sylvian electrodes in 14 patients, the superior frontal electrodes $(\mathrm{F} 3 / \mathrm{F} 4)$ in six patients, and the posterior temporal electrodes in five patients. The incidence of discharges on the scalp varied from 0.4 to 21 discharges $/ \mathrm{min}$ (mean 3.93 (SD 4.17)).

\section{ANALYSIS OF ELECTROCORTICOGRAPHIC}

DISCHARGES

The preactivation time varied between 120 and 440 seconds (mean 4.7 (SD 1.38) minutes). We identified a total of $25 \mathrm{ECoG}$ patterns. In nine patients $(56.25 \%)$ we found two independent patterns. We distinguished four different subtemporal patterns, eight lateral temporal, seven mixed (subtemporal, lateral temporal, and, in one patient, suprasylvian), and one orbital. Lateral temporal patterns were seen in 11 patients, mixed patterns in seven patients, subtemporal patterns in six patients, and an orbital pattern in one patient. The dominant electrodes were subtemporal in 10 patterns (from 10 patients), lateral temporal in 14 patterns (from 13 patients), and orbital in one pattern (from one patient). In one pattern from one patient, dominant electrodes were located on the subtemporal and lateral temporal cortices. The subtemporal electrodes were involved in the discharges but were nondominant in three patterns (from three patients), the lateral temporal electrodes in four patterns (from four patients), and the suprasylvian electrodes in one pattern (from one patient). In four patients the spikes were only negative and in 12 patients spikes showed a subtle positive initial deflection followed by a much larger negative peak. Four patients showed positive spikes. The three types of spikes (negative, positive-negative, and positive) were detected in four patients and all patients with positive spikes also showed negative or positive-negative spikes. Only one patient showed a higher incidence of positive than of negative or positive-negative spikes. Positive spikes never involved more than two contiguous electrodes. The incidence of discharges varied from 0.6 to 14 discharges $/ \mathrm{min}$ (mean 6.1 (SD 4.05)). In two patients some spikes were of complex polyphasic morphology. Three patients showed runs of rhythmic multiple spikes (polyspikes) at 20-22 cycles/s,

Table 1 Relation of topography of the dominant electrode(s) on the scalp with presence of spikes (regular font) and with topography of dominant electrodes (bold font) on the ECoG

\begin{tabular}{|c|c|c|c|c|c|c|c|c|c|c|c|}
\hline \multirow[b]{2}{*}{ Scalp DE } & \multicolumn{3}{|c|}{ Subtemporal } & \multicolumn{6}{|c|}{ Lateral temporal } & \multirow[b]{2}{*}{ SS } & \multirow[b]{2}{*}{$O b$} \\
\hline & Ant & Mid & Post & $S a$ & $S p$ & $M a$ & $M p$ & Ia & $I p$ & & \\
\hline \multirow[t]{2}{*}{ No } & 10 & 13 & 7 & 9 & 5 & 7 & 8 & 8 & 7 & 1 & 1 \\
\hline & 3 & 9 & 2 & 4 & 2 & 5 & 5 & 0 & 1 & 0 & 1 \\
\hline $\mathrm{T} 1 / \mathrm{T} 2$ & 8 & 10 & 5 & 8 & 5 & 7 & 7 & 7 & 6 & 1 & 1 \\
\hline \multirow[t]{3}{*}{$\mathrm{n}=13$} & 80 & 76.9 & 71.4 & 88.9 & 100 & 100 & 87.5 & 87.5 & 85.7 & 100 & 100 \\
\hline & 2 & 6 & 1 & 4 & 2 & 5 & 4 & 0 & 1 & 0 & 1 \\
\hline & 66.7 & 66.6 & 50 & 100 & 100 & 100 & 80 & & 100 & & 100 \\
\hline $\mathrm{A} 1 / \mathrm{A} 2$ & 2 & 4 & 2 & 2 & 0 & 2 & 3 & 2 & 2 & 0 & 1 \\
\hline \multirow[t]{3}{*}{$\mathrm{n}=5$} & 20 & 30.8 & 28.6 & 22.2 & & 28.6 & 37.5 & 25 & 28.6 & & 100 \\
\hline & 1 & 2 & 1 & $\mathbf{0}$ & 0 & 1 & 3 & 0 & 0 & 0 & 1 \\
\hline & 33.3 & 22.2 & 50 & & & 20 & 60 & & & & 100 \\
\hline \multirow{3}{*}{$\begin{array}{l}\mathrm{T} 3 / \mathrm{T} 4 \\
\mathrm{n}=1\end{array}$} & 1 & 1 & 0 & 0 & 0 & 0 & 0 & 0 & 0 & 0 & 0 \\
\hline & 10 & 7.7 & & & & & & & & & \\
\hline & 1 & 1 & $\mathbf{0}$ & 0 & 0 & 0 & 0 & 0 & 0 & 0 & 0 \\
\hline \multirow{3}{*}{$\begin{array}{l}\mathrm{F} 3 / \mathrm{F} 4 \\
\mathrm{n}=1\end{array}$} & $\begin{array}{l}33.3 \\
0\end{array}$ & $\begin{array}{l}11.1 \\
1\end{array}$ & 1 & 0 & 0 & 0 & 1 & 0 & 0 & 0 & 0 \\
\hline & & 7.7 & 14.3 & & & & 12.5 & & & & \\
\hline & 0 & $\begin{array}{l}1 \\
11.1\end{array}$ & 0 & 0 & 0 & 0 & 0 & 0 & 0 & 0 & 0 \\
\hline T5/T6 & 2 & 2 & 2 & 2 & 1 & 1 & 1 & 2 & 2 & 1 & 0 \\
\hline \multirow[t]{3}{*}{$\mathrm{n}=2$} & 20 & 15.4 & 28.6 & 22.2 & 20 & 14.3 & 12.5 & 25 & 28.6 & 100 & \\
\hline & 0 & 1 & 1 & $\mathbf{0}$ & $\mathbf{0}$ & 1 & 1 & 0 & 1 & 0 & 0 \\
\hline & & 11.1 & 50 & & & 20 & 20 & & 100 & & \\
\hline
\end{tabular}

The left most column shows the topography of dominant electrodes on the scalp. The other columns show the number (non-italics) and proportion (italics) of patients showing spikes (regular font) and largest spikes (bold font) at the corresponding topographies on the ECoG. The top rows of numbers (No) represent the number of patients presenting spikes (regular font) or largest spikes (bold font) at the corresponding location on the ECoG. The rest of the cells show the number of patients presenting scalp discharges with a dominant electrode indicated on the left column and presenting ECoG spikes (regular) or largest ECoG spikes (bold) at the topographies indicated by the lables on the top. The numbers in italics indicate the same figure as a proportion of the total number of patients presenting spikes (or largest spikes) at the corresponding location in the ECoG (top row of numbers). For instance, among the patients with dominant electrodes at T1/T2, eight patients showed spikes in the anterior aspect of the superior temporal gyrus (representing $88.9 \%$ of all patients showing spikes in this region on the ECoG), and only five in the posterior aspect of the same gyrus (representing $100 \%$ of all patients showing spikes in this region). In other words, $88.9 \%$ of patients showing spikes in the anterior aspect of the superior temporal region and all patients showing spikes in the posterior aspect of the superior temporal region had largest spikes at $\mathrm{T} 1 / \mathrm{T} 2$ on the scalp. $\mathrm{DE}=$ dominant electrode on the scalp; ant=anterior subtemporal; mid=midsubtemporal; pos=posterior subtemporal; $\mathrm{Sa}=$ anterior aspect of the superior temporal gyrus; $\mathrm{Sp}=$ posterior aspect of the superior temporal gyrus; $\mathrm{Ma}=$ anterior aspect of the mid temporal gyrus; $\mathrm{Mp}=$ posterior aspect of the mid temporal gyrus; Ia= anterior aspect of the inferior temporal gyrus; Ip=posterior aspect of the inferior temporal gyrus; $\mathrm{SS}=$ suprasylvian; $\mathrm{Ob}=$ orbital. 
Table 2 Duration of spikes on ECoG and scalp recordings, and duration of modelled (calculated) scalp spikes from ECoG spikes

\begin{tabular}{|c|c|c|c|c|c|c|c|c|c|}
\hline \multirow[b]{2}{*}{ Patient } & \multicolumn{3}{|c|}{ Duration ECoG } & \multicolumn{3}{|c|}{ Duration scalp } & \multicolumn{3}{|c|}{ Duration modelled scalp } \\
\hline & $n$ & Mean & $S D$ & $n$ & Mean & $S D$ & $n$ & Mean & $S D$ \\
\hline 1 & 47 & $53.0^{\star}$ & 15.9 & 14 & 88.8 & 21.9 & & & \\
\hline 2 & 10 & $43.0^{\star}$ & 13.9 & 62 & 82.9 & 19.8 & & & \\
\hline 3 & 15 & $41.9^{\star}$ & 12.3 & 6 & 84.6 & 19.7 & & & \\
\hline 4 & 14 & $58.9^{\star}$ & 24 & 5 & 86.4 & 18.6 & 6 & 72 & 13.7 \\
\hline 5 & 23 & $55.3^{\star}$ & 13.4 & 23 & 68.8 & 15.3 & 20 & 64.2 & 14.8 \\
\hline 6 & 23 & $65.3^{\star}$ & 21.4 & 18 & 107.8 & 28.7 & $\begin{array}{l}15 \\
5\end{array}$ & $\begin{array}{l}74.4^{\star} \\
105.6(\ln )\end{array}$ & $\begin{array}{l}26.2 \\
19.2\end{array}$ \\
\hline 7 & 13 & $46.6^{\star}$ & 19.3 & 7 & 106.4 & 34.1 & & & \\
\hline 8 & 21 & $54.9^{\star}$ & 19.5 & 40 & 97.0 & 24.1 & & & \\
\hline 9 & 12 & $45.6^{\star}$ & 20.3 & 10 & 69.0 & 23.0 & 9 & 55.3 & 12.65 \\
\hline 10 & 47 & $\begin{array}{l}65.6^{\star} \\
65.6^{\star}\end{array}$ & $\begin{array}{l}17.5 \\
17.5\end{array}$ & $\begin{array}{l}31 \\
13\end{array}$ & $\begin{array}{l}40.2(\mathrm{~F} 3) \\
88.0(\mathrm{~A} 1)\end{array}$ & $\begin{array}{l}15.4 \\
19.9\end{array}$ & & & \\
\hline 11 & 22 & $45.0^{\star}$ & 15.1 & 13 & 76.6 & 23.1 & & & \\
\hline 12 & 32 & $60.7^{\star}$ & 16.4 & 10 & 74.5 & 15.6 & 9 & 78.6 & 18.8 \\
\hline 13 & 22 & 56.3 & 23.2 & 23 & 60.0 & 17.2 & & & \\
\hline 14 & 53 & $55.7^{\star}$ & 18.1 & 18 & 72.3 & 27.1 & 25 & 78.8 & 21.7 \\
\hline 15 & 8 & $49.0^{\star}$ & 11.5 & 70 & 100 & 43.9 & & & \\
\hline 16 & 63 & $37.0^{\star}$ & 14.5 & 116 & 76.9 & 28.8 & 11 & $50.7^{\star}$ & 10.5 \\
\hline
\end{tabular}

*Significant differences with the duration of measured scalp spikes. Most patients show significant differences between ECoG spikes and measured scalp spikes. However, most patients with calculated scalp spikes show no differences between measured and modelled scalp spikes. $n=$ Number of discharges; $l n=$ considering spikes restricted to the lateral neocortex.

restricted to one channel, but this activity was not considered in the analysis.

\section{CORRELATION BETWEEN ECOG AND SCALP}

RECORDINGS

Among the nine patients that showed two ECoG patterns only one patient showed two scalp patterns. Therefore, in eight patients, two discharge patterns were seen in the ECoG whereas only one pattern was seen on the scalp. In 13 patients at least one ECoG pattern had a higher incidence than scalp patterns. The remaining three patients showed an incidence of discharges higher on scalp recordings than in the ECoG. The polarity of spikes was always negative or positive-negative on scalp recordings and negative, positive-negative, or positive polarities were seen on the ECoG recordings.

Table 1 shows the relation between the location of the dominant electrode(s) of the scalp discharges and the presence of ECoG spikes at different locations (regular font). There does not seem to be any clear relation between the topography of the dominant electrodes on the scalp and the absence or presence of spikes in specific cortical locations in the ECoG. In particular, it does not seem that when the dominant scalp electrodes are more anterior (T1/T2) or posterior (A1/A2, T5/T6), ECoG spikes are similarly located.

Table 1 also shows the relation in the topography of dominant electrodes on the scalp and in ECoG recordings (bold font). It seems that the most frequent dominant electrode in subtemporal discharges tends to be located on the midsubtemporal region, irrespective of the location of the dominant electrode on the scalp. A slightly clearer correlation was found between the topography of dominant electrodes on scalp and on the lateral neocortex. However, although patients with the largest spikes at the anterior temporal electrode (T1/T2) often have largest spikes on the anterior lateral neocortex, they can also often show the largest spikes in the posterior lateral neocortex.
In all 15 patients who showed a single scalp pattern, the mean duration of spikes was longer on scalp than on ECoG (table 2) and this difference was statistically significant in 14 patients. The patient who had two scalp patterns showed a mean duration of spikes longer on scalp EEG than on ECoG for one pattern (T1, A1, T3, T5) and shorter for the other pattern $(\mathrm{F} 3, \mathrm{Fz})$.

SPATIAL AVERAgING

Fifteen digitised ECoG recordings were analysed. Four patients showed ECoG discharges mainly restricted to one channel and it was therefore not possible to model scalp spikes. One patient showed only complex polymorphic ECoG discharges and modelling of scalp spikes was not attempted. Three patients had scanty epileptiform discharges on the ECoG, showing less than five propagated discharges in the preactivation ECoG. Thus, in seven patients we were able to obtain five or more modelled spikes for statistical comparison. In five patients the durations of measured and modelled scalp spikes were not statistically different. In the remaining two patients, modelled spikes showed statistically significant shorter duration than measured scalp spikes. In one of these two patients, these differences disappeared when scalp spikes were modelled from lateral temporal ECoG spikes, excluding subtemporal spikes. In the other patient, ECoG spikes were confined to one or two adjacent recording channels which showed negligible latency differences.

\section{Discussion}

This study confirms that ECoG recordings show more complexity and variability than scalp EEG tracings. Generally, ECoG recordings showed a greater number of topographic patterns and higher frequency of discharges than scalp EEGs. It also seems that ECoG discharges can be more localised than scalp discharges and, if they are widespread, their waveform is exceedingly variable among corti- 
cal regions. We found that isolated discharges, restricted to one electrode, are common in corticographic recordings whereas scalp discharges tend to affect two or more electrodes. If we consider that ECoG electrodes are only 1-2 $\mathrm{cm}$ apart and scalp electrodes can be up to 4-5 $\mathrm{cm}$ apart, our findings confirm the suggestion by Cooper $e t a l^{11}$ that only the most widespread ECoG discharges are seen on the scalp. This is also consistent with our findings which show that ECoG discharges repeatedly exhibit higher incidence than scalp discharges, suggesting that some ECoG discharges are not seen on the scalp. Even under this assumption, widespread ECoG discharges are still more localised than scalp discharges. Whereas ECoG discharges tended to be restricted to the temporal lobe, scalp discharges often extended as far as the prefrontal electrode ( $\mathrm{Fp} 1 / \mathrm{Fp} 2)$, located over the pole of the frontal lobe. This is a consistent finding that has been previously reported ${ }^{12}$ and has been explained by propagation of discharges to the frontal lobe. This explanation, however, is unlikely, as orbital or suprasylvian frontal involvement in corticographic recordings was seen only in two patients. An alternative explanation is that the topography of electrical fields recorded on the scalp is the result of a significant distortion of neuronal electrical fields by the spatial distribution of the CSF, brain coverings, skull, and scalp. This also explains why, in $50 \%$ of patients the number of patterns seen on the scalp is smaller than the number of discharge patterns on ECoG. If the topography of fields on the scalp depends largely on the physical characteristics of brain coverings, which are constant for each patient and similar between patients, relatively different discharge patterns on the ECoG will be seen with similar topography on the scalp. This is indeed suggested by our finding that eight of the nine patients with two discharge patterns on the ECoG show a single pattern on the scalp and, if all patients are pooled, markedly different ECoG patterns appeared as scalp discharges with maximal amplitude at the anterior temporal electrode (T1/T2).

SPIKE POLARITY

Positive spikes were never seen on the scalp but found in $25 \%$ of ECoGs. No active or rectifying elements are thought to exist across the brain coverings that could reverse field polarity. However, positive ECoG spikes always showed a very localised topography, involving only one or two adjacent electrodes and, as such localised discharges do not seem to reach the scalp, it is not surprising that positive spikes were not seen in the EEG.

\section{SPIKE DURATION}

Another interesting finding is that scalp spikes are significantly longer in duration than spikes on the ECoG. This could be explained by changes associated with tissue capacitance or by the existence of latency differences among ECoG spikes. It can be shown that a homogeneous poorly conducting medium, having resistive and capacitive properties, behaves as a resistor in parallel with a capacitor, with a time constant equal to permitivity/conductivity. ${ }^{13}$ For bone, for instance, which is the least conductive tissue, the measured values for relative permitivity $(10000)$ and conductivity $(0.01 \mathrm{~S} / \mathrm{m})$ at $10 \mathrm{~Hz}^{14}$ would be compatible with a time constant of $8.85 \mu$ s which is far below the time resolution of EEG sampling frequencies (usually $<300 \mathrm{~Hz}$ ). Thus time differences of the order of several milliseconds, found among spikes in intracranial recordings, including ECoGs, cannot easily be explained by capacitive effects and have been previously described and interpreted in terms of neuronal propagation and conduction time. ${ }^{5}{ }^{10}$ Spatial averaging due to distance and the effects of extracerebral layers can result in increased duration of scalp spikes, as averaging of several sharp spikes having small latency differences will generate longer lasting and blunter spikes. Such mild desynchronisation of ECoG spikes is likely to be a significant contributing factor, as we have been able to simulate the duration of scalp spikes by averaging ECoG spikes in six of the seven patients who showed sufficiently frequent and widespread ECoG discharges (table 2). As a practical application of our hypothesis, it can be suggested that more widespread neuronal activation would result in longer propagation time and more desynchronised ECoG spikes, generating longer duration spikes on the scalp, perhaps in addition to more widespread scalp topography.

SPATIAL DISTRIBUTION

It is intriguing that most electrocorticographic patterns appeared on the scalp with largest amplitude at the anterior temporal electrode (T1/T2), not only because it means that quite different ECoG patterns are associated with similar maxima on the scalp, but also because the anterior temporal electrode lies further away from the temporal lobe than other scalp electrodes. It could be argued that the orientation of neuronal sources would generate a maximum at the anterior temporal electrode but this is improbable given the variability of discharge distribution in the ECoG records (table 1). A more probable explanation is that the anterior temporal maxima in scalp recordings result from the properties of extracerebral layers. Two physical properties of extracerebral tissue can theoretically distort electric fields and generate an anterior temporal maximum: the presence of tissue inhomogeneities or of high conductivity pathways close to the anterior temporal electrodes.

\section{Inhomogeneities}

The anterior temporal electrode lies over a rather inhomogeneous region composed of the temporal muscle, the thin greater wing of the sphenoid bone, and the cisterna ambiens. Such inhomogeneities can generate complicated layers of different conductivities and capacitance. As the presence of conductivity boundaries usually results in a greater signal attenuation than if there were no boundaries, ${ }^{15}$ inhomogeneities in conductivities are unlikely to explain the larger electric fields seen in this region. 
Alternatively, inhomogeneities in capacitance of the tissues could explain the accumulation of charges in this region. This however, also seems improbable, because at the EEG frequencies, conductivity rather than capacitance largely determines the impedance of biological tissues to external electric fields and the effects of inhomogeneities in capacitance are probably small unless inhomogeneities are very large.

High conductivity pathways

The second physical property that could explain a predominant anterior temporal maximum is that a significant proportion of the signal reaches the scalp through pathways of higher conductivity located anteriorly. Because the bone is the tissue with lowest conductivity, such high conductivity pathways are likely to exist where there are holes in the skull. There are several holes located anteriorly (the optic foramen, foramen ovale, and the superior orbital fissure) and relatively close to anterior temporal scalp electrodes. Such holes are filled with vascular structures and nerves which possess fairly high conductivities. Indeed Kanner and Jones ${ }^{16}$ have tentatively hypothesised that one of the advantages of suitably located sphenoidal electrodes is the absence of interposed bone (being located near a skull hole). As the resistivity of bone is between 25 and 100 times higher than the resistivity of blood or nervous tissue, ${ }^{17}{ }^{18}$ a discharge will probably have to be over 20 times bigger posteriorly to be seen with largest amplitude at the midtemporal or posterior temporal electrode on the scalp, provided that the magnitude of the areas having different conductivities is disregarded. Once most of the signal has left the skull at specific points, it would be conducted by the highly conductive extracranial tissues resulting in a quasiequipotential field over the scalp. This would explain why scalp discharges show no change in waveform over a wide distribution and are often seen at the prefrontal electrode on the scalp without involvement of the frontal lobe in the corticography.

Conduction of electric (and magnetic) fields through brain coverings and scalp has often been modelled as concentric layers of homogeneous materials showing different conductivities. ${ }^{19-21}$ If our last hypothesis is correct, discontinuities in some conductive layers should be included, as these seem to be main determinants of the topography of scalp signals. In fact, recent theoretical models have shown the dramatic effects of skull holes on the topography of scalp signals. ${ }^{22} \mathrm{~A}$ direct consequence of this hypothesis is that spikes seen near the foramen ovale (for instance, by sphenoidal electrodes) will tend to have the same waveform but larger amplitudes than spikes recorded on the scalp. Indeed, the comparison of simultaneous recordings from scalp and sphenoidal electrodes shows that this is the case. $^{23}$ When multicontact sphenoidal electrodes are used, the deepest contact, located outside the skull but nearest to the foramen ovale, records larger spikes than the other sphenoidal contacts or scalp electrodes, including the anterior temporal electrode. The larger amplitude of spikes seen at the sphenoidal electrode nearest to the foramen ovale cannot be explained solely on the basis of closeness of this electrode to mesial temporal neural generators, because spikes seen at mesial temporal structures are commonly not seen on the scalp $^{5}$ whereas the vast majority of spikes seen at the sphenoidal electrode, located extracranially, are also recorded at the anterior temporal scalp electrode. ${ }^{23}$ This interpretation is supported by Krauss et $a l^{24}$ who found that anterior cheek electrodes are as effective as sphenoidal electrodes in the identification of ictal activity. Similarly, if a substantial amount of EEG signal reaches the scalp through orbital holes, orbital electrodes should be as effective as anterior temporal electrodes in the identification of interictal discharges despite being located even further away from the temporal lobe. This has indeed been recently reported by Gambardell et $a l^{25}$ for lateral orbital electrodes.

\section{LIMITATIONS}

The study of the transmission of electric fields through brain coverings is complicated by the fact that extensive electrode coverage of brain and scalp cannot be simultaneously obtained without significant disruptions of brain coverings. Methods can easily alter the structures the effects of which ought to be studied and non-simultaneous recordings of scalp and ECoG were therefore required to obtain detailed topographical information of cortical discharges and scalp EEG with intact brain coverings. Likewise, it would have been desirable to record scalp discharges also under general anaesthesia but this would have lengthened the surgical procedure unacceptably. The finding that discharge duration on the scalp can be predicted from ECoG discharges suggests that anaesthesia has not induced a significant bias. The effects of anaesthesia have been minimised by comparing ECoG with scalp records obtained during sleep. In our experience, spikes seen with chronic intracranial and simultaneous scalp recordings in unanaesthetised patients obtained during presurgical assessment also appear sharper, steeper, and shorter than scalp discharges, as indeed has been reported by others, ${ }^{34}$ suggesting that the effects of anaesthesia are probably not significant.

\section{Conclusion}

In summary, it seems that, in temporal lobe epilepsy, scalp discharges originating from widespread ECoG discharges in the temporal lobe tend to appear with relatively similar patterns on the scalp, with largest amplitudes in the anterior temporal region. This is probably due to local anatomical peculiarities in the brain coverings, such as skull holes, rather than to the location of neuronal generators within the temporal lobe. Positive spikes were always restricted to one or two electrodes in the ECoG which would explain their absence in scalp recordings. Widespread neuronal propagation resulting in latency differences between cortical spikes can be responsible for the generation of longer duration and blunter spikes on the scalp. Thus more widespread 
neuronal activation would result in longer duration of spikes on the scalp in addition to a more widespread scalp topography. These findings should be considered when interpreting scalp EEG in routine clinical practice and for source localisation.

JLFT has been supported by Instituto de Salud "Carlos III" (Fondo de Investigación Sanitaria, FIS 97/5112 and 98/5008, Spain). We are grateful to Dr David Holder for his helpful discussion on the interpretation of the results.

1 Niedermeyer E. Epileptic seizure disorders. In: Niedermeyer Lopes da Silva F, eds. Electroencephalography: basic principles, clinical applications and related fields. 2nd ed Baltimore: Urban and Schwarzenberg, 1987:465.

2 Abraham K, Ajmone Marsan C. Patterns of cortical discharges and their relation to routine scalp electroencephalography. Electroencephalogr Clin Neurophysiol 1958; 10:447-61.

3 Spencer SS, So NK, Engel J Jr, et al. Depth electrodes. In: Engel J Jr, ed. Surgical treatment of the epilepsies. 2nd ed. New York: Raven, 1993:359-76.

4 Ajmone Marsan C. Chronic intracranial recording and electrocorticography. In: Daly DD, Pedley TA, eds. Current practice of clinical electroencephalography. 2 nd ed. New York: practice of clinical electroe

5 Alarcón G, Guy CN, Binnie CD, et al. Intracerebral propaAlarcon G, Guy CN, Binnie CD, et al. Intracerebral propagation of interictal activity in partial epilepsy: implications
for source localisation. 7 Neurol Neurosurg Psychiatry 1994; for source 1

6 Polkey CE, Binnie CD. Neurosurgical treatment of epilepsy. In: Laidlaw J, Richens A, Chadwick D, eds. A textbook of epilepsy. 4th ed. Edinburgh: Churchill Livingstone, 1993 561-611.

7 Margerison JH, Binnie CD, McCaul IR. Electroencephalographic signs employed in the location of rupture intracranial arterial aneurysms. Electroencephalogr Clin Neurophysiol 1970;28:296-306.

8 Silverman D, Sannit T, Ainspac S, et al. The anterior temporal electrode and the ten-twenty system. Electroencephalogr Clin Neurophysiol 1960;12:735-7.

9 Chatrian GE, Bergamini L, Dondey M, et al. A glossary of terms most commonly used by clinical electroencephalog-
raphers. Electroencephalogr Clin Neurophysiol 1974;37:538raph.

10 Alarcón G, García Seoane JJ, Binnie CD, et al. Origin and propagation of interictal discharges in the acute electrocorticogram: implications for pathophysiology and
surgical treatment of temporal lobe epilepsy. Brain 1997;120:2259-82.

11 Cooper R, Winter AL, Crow HJ, et al. Comparison of subcortical, cortical and scalp activity using chronically indwelling electrodes in man. Electroencephalogr Clin Neurophysiol 1965;18:217-28.

12 Emerson RG, Turner CA, Pedley TA, et al. Propagation patterns of temporal spikes. Electroencephalogr Clin Neurophsysiol 1995;94:338-48.

13 Cheng DK. Field and wave electromagnetics. 2nd ed. Reading: Addison-Wesley, 1989:215-16.

14 Kosterich JD, Foster KR, Pollack SR. Dielectric permittivity and electrical conductivity of fluid saturated bone. IEEE Trans Biomed Eng 1983;30:81-6.

15 Gloor P Neuronal generators and the problem of localization in electroencephalography: application of volume conductor theory to electroencephalography. $\mathcal{f}$ Clin Neuroductor theory to electro

16 Kanner AM, Jones JC. When do sphenoidal electrodes yield additional data to that obtained with antero-temporal elecrodes? Electroencephalogr Clin Neurophysiol 1997;102:12-

17 Geddes LA, Baker LE. The specific resistance of biological material: a compendium of data for the biomedical engineer and physiologist. Medical and Biological Engineering 1967;5:271-93.

18 Law SK. Thickness and resistivity variations over the upper surface of the human skull. Brain Topogr 1993;6:99-109.

19 Geselowitz D. On bioelectric potentials in an inhomogeneous volume conductor. Biophys f 1967;7:1-11.

20 Sarvas J. Basic mathematical and electromagnetic concepts of the biomagnetic inverse problem. Phys Med Biol 1987;32:11-22.

21 de Munck JC. The potential distribution in a layered anisotropic spheroidal volume conductor. $\mathcal{F}$ Appl Physiol 1988;64:464-70

22 van den Broek SP, Reinders F, Donderwinkel M, et al. Volume conduction effects in EEG and MEG. Electroenceph Clin Neurophysiol 1998;106:522-34.

23 Binnie CD, Marston D, Polkey CE, et al. Distribution of temporal spikes in relation to the sphenoidal electrode. Electroenceph Clin Neurophysiol 1989;73:403-9.

24 Krauss GL, Lesser RP, Fisher RS, et al. Anterior cheek electrodes are comparable to sphenoidal electrodes for the identification of ictal activ

25 Gambardella A Pucci F, Le Piane E et al. Usefulness of Pate cor activity: a study of 60 patients with complex partial seizures. Electroencephalogr Clin Neurophsysiol 1998,107: 